\title{
Matthew L. Carlsson, Michael J. Link, Colin L.W. Driscoll, et al. (eds): Comprehensive management of vestibular schwannoma
}

\author{
2019 Hardback, pp.: 593, 441 illustrations
}

Torstein R. Meling ${ }^{1}$ (D)

Received: 27 December 2019 / Accepted: 31 December 2019 /Published online: 8 January 2020

(C) Springer-Verlag GmbH Austria, part of Springer Nature 2020

A neurosurgical textbook must reflect contemporary knowledge, live up to its title, and clearly define its target audience, be well organized and concisely written. The book should be well illustrated, with good diagrams, tables, illustrations, and photos and perhaps even video access. Lastly, it should be authored by knowledgeable, active practitioners of our specialty and not champions of the past.

As its name implies, the chief objective of "Comprehensive Management of Vestibular Schwannoma" was to "create a comprehensive clinical textbook covering all aspects of vestibular schwannoma management." The target audiences are trainees (residents and fellows) and practicing physicians (early, as well as mid- to later-career attending physicians) who care for patients with vestibular schwannoma.

The book totals 556 pages (excluding Index) and is subdivided into 10 parts, with a total of 84 chapters. It contains 441 color illustrations, dissections, clinical images, and line drawings. In Part I, history, epidemiology, histopathology, and molecular biology are presented (27 pages), whereas Part II deals with the clinical presentations and evaluations (35 pages). Part III presents an overview of vestibular schwannoma management (12 pages) and is followed by chapters on conservative management (Part IV; 6 pages), radiation (Part V; 67 pages), and microsurgery (Part VI; 185 pages). Over 11 chapters, the principles of radiobiology are explained, followed by chapters on Gamma Knife ${ }^{\circledR}$ radiosurgery, LINAC, CyberKnife $\AA$, and proton beam radiotherapy, as well as on controversies such as SRS or RT for large vestibular schwannomas. Over 24 chapters, all aspects of microsurgery for vestibular schwannomas are

Torstein R. Meling

torsteinrmeling@gmail.com

1 Geneva University Hospital, Rue Gabrielle Perret-Gentil 4, 1211, Geneva 14, Switzerland covered, including neuroanesthesiology, monitoring, training, microsurgical anatomy, all the different approaches, complication avoidance, and postoperative care. Part VII presents outcomes (67 pages), Part VIII rehabilitation (74 pages), and Part IX controversies (29 pages). Finally, Part X deals with neurofibromatosis type 2 (40 pages).

"Comprehensive Management of Vestibular Schwannoma" is contemporary and comprehensive. It is written by experts including neurosurgeons, otolaryngologists, radiation oncologists, neurologists, neuroradiologists, and audiologists from the Mayo Clinic or by renowned international faculty.

The subdivisions are logical and annotated by clear headings and subheadings. The chapters are generally well balanced, with a mean of 5 pages (including references). The most extensive chapter, "Anatomy of Vestibular Schwannoma Surgery" by Dr. Rhoton, runs over 19 pages. Chapter summary tables provide readers with a rapid clinical reference derived from the published world literature.

As with any multi-authored book, there are some redundancies with overlapping text, as well as some internal discrepancies. However, the editors and Thieme have succeeded in getting a uniform presentation by making the contributions adhere to their format.

The quality is generally consistent from subject to subject, perhaps with the exception of part VIII Medical and surgical rehabilitation, where particularly the chapters on facial reanimations expose a lack of scientific evidence-base and long-term outcome studies.

In conclusion, I find the book concise, well written, and beautifully illustrated. The authors present all management modalities in a balanced manner. The part on controversial or challenging aspects of care is particularly contemporary and "Comprehensive Management of Vestibular Schwannoma" succeeds in providing great value to a target audience that ranges from the novice to the expert. 